\title{
Prognostic Value of Intraoperative Pleural Lavage Cytology as an Independent Prognostic Factor in Non- Small Cell Lung Cancer: A Retrospective Study
}

\section{Sirawee Ekkasak}

Ramathibodi Hospital

Pisut Pongchaikul

Ramathibodi Hospital

Montien Ngodngamthaweesuk ( $\sim$ montien.ngo@mahidol.ac.th )

Ramathibodi Hospital

\section{Research Article}

Keywords: non-small cell lung cancer, pleural lavage cytology, intraoperative pleural lavage

Posted Date: May 28th, 2021

DOl: https://doi.org/10.21203/rs.3.rs-537686/v1

License: () (1) This work is licensed under a Creative Commons Attribution 4.0 International License. Read Full License 


\section{Abstract}

\section{Background}

The aim of this study is to demonstrate that intraoperative PLC has a role in predicting clinical outcomes in NSCLC patients.

\section{Methods}

Intraoperative PLC was performed in NSCLC patients who had no pleural effusion before the operation. PLC was performed three times for each patient. PLC1 was performed after the thoracotomy; PLC2 was performed immediately after complete operation; and PLC3 was performed after complete operation and washed the pleural cavity with $5,000 \mathrm{ml}$ of normal saline solution. Clinical records of 178 patients in Ramathibodi Hospital from 2012 to 2016 were retrospectively reviewed and analysed for the relevance of intraoperative PLC and clinical outcomes.

\section{Results}

178 patients were included in this study; 67 patients were male (37.6\%). Metastatic tumour from primary lung cancer occurred in 56 patients (31.4\%). Positive intraoperative PLC was significantly associated with higher metastatic rate $(p<0.05)$. Survival rate in the positive intraoperative PLC group was significantly worse than that in the negative PLC group $(p<0.05)$.

\section{Conclusions}

This study shows positive intraoperative PLC was statistically significant for increasing metastatic rate and decreasing survival rate in NSCLC patients. Intraoperative PLC could provide important information for the prediction of disease progression and treatment planning.

\section{Background}

Lung cancer is the third most common cancer in Thailand[1-3]. Amongst them, NSCLC is the most common type, contributing $80-85 \%$. The current diagnosis and staging system for NSCLC is based on the TNM system, which is defined by using tumour size, lymph node metastasis, and distant metastasis. The TNM staging system is efficiently used for determining disease prognosis and treatment options. The overall five-year survival rate for NSCLC is $24 \%$. However, the survival rate depends on several factors, especially the stages of disease. For patients with stage $1 \mathrm{~A}$ disease, the overall five-year survival rate is more than $90 \%$, whereas it is less than $10 \%$ for stage 4 patients. A different stage leads to a different treatment. In the early stage NSCLC, upfront surgery is the treatment of choice while chemoradiation is preferred in metastatic patients. In NSCLC, pleural effusion, especially malignant pleural effusion, demonstrates an advanced stage of NSCLC.

Pleural effusion with hemorrhagic or serosanguinous appearance is more likely to be malignant. In biochemical analysis, malignant pleural effusion is usually exudate. The simplest method to diagnose malignant pleural effusion is the presence of malignant cells from pleural fluid cytology. Another method is to demonstrate tumour cells in the pleural tissues which can be obtained from pleural biopsy. According to the TNM staging system for NSCLC, patients with malignant effusion are categorised as M1a or stage 4 disease [4]. However, pleural effusion 
is only present in $7-23 \%$ of lung cancer patients and malignant pleural effusion is found in only $20 \%$ of patients with an advanced stage of disease [5,6]. Several studies show that cancer cells can be detected in the thoracic cavity from pleural lavage, even in the absence of pleural effusion $[7,8]$.

IPL is the procedure done by using physiologic saline solution irrigate in the thoracic cavity over the lung surface. In our study, we performed 3 IPLs for each patient and sent specimens for cytologic analysis. Interestingly, IPL cytology has been reported to be associated with clinical outcomes in many studies [9-12]. Nevertheless, IPL cytology is not included in current methods for diagnosis and prognosis prediction [13]. We demonstrated here to determine whether intraoperative PLC can reveal micro metastasis and assess the prognostic significance in clinical outcomes.

\section{Patients And Methods}

\section{Patients}

This retrospective study was approved by the Ethics Committee of the Institute (MURA2017/695; ID 10-60-19) and compliance with Helsinki declaration. The need of informed consent was waived by ethical committee of Faculty of Medicine Ramathibodi Hospital, Mahidol University, Thailand. No identifying information was recorded by research team. We included all patients with NSCLC who underwent lung surgery and intraoperative pleural lavage in Ramathibodi Hospital from January 2012 to December 2016. Patients who had preoperative pleural effusion and patients whose data had been lost were excluded from this study. The final study sample consisted of 178 patients.

\section{Procedure}

We performed intraoperative PLC three times for each patient. The $1^{\text {st }}$ PLC (PLC 1) was performed after the thoracotomy. The $2^{\text {nd }}$ PLC (PLC 2) was performed after complete the operation and the $3^{\text {rd }}$ PLC (PLC 3) was performed after complete the operation and carefully washed the pleural cavity with $5,000 \mathrm{ml}$ of normal saline solution. $500 \mathrm{ml}$ of pleural lavage fluid for each specimen was collected and sent for cytologic examination. The cytologic report of malignant cells or atypical cells was considered to be positive. The cytologic result came back within 2 weeks after the operations. PLC technique and surgical procedure were standardised in all patients and a single-surgeon surgeries were performed throughout the study. Pathological staging was performed by using the International Staging System for lung cancer[13].

\section{Statistical analysis}

Continuous data in this study were reported as mean with SD or median with IQR. The difference between mean or media was conducted using the independent t-test or the Mann-Whitney U-test. Categorical variables were presented and analysed by chi-squared test or Fisher's exact test. The survival and recurrent rate were analysed by Kaplan-Meier method. The differences between the survival curves were assessed by using the log-rank test. Statistical analysis was performed in STATA version 14 (StataCorp. 2015. Stata Statistical Software: Release 14. College Station, TX: StataCorp LP.). P values of $<0.05$ were considered statistical significance in all analyses.

\section{Results}




\section{Patient characteristics}

From 178 patients in total, 67 patients were male (37.6\%). Intraoperative PLC was positive in 20 cases (11.23\%). Patient baseline characteristics were not different between the recurrent/metastatic group and non-recurrent group. However, visceral pleural invasion, vascular invasion, lymphatic invasion and post-operative staging were higher in recurrent/metastatic group (Table 1). Similar results were reported when we analysed patient baseline characteristics in death and non-death groups (Table 2).

\section{Pleural lavage cytology is associated with mortality}

Patients with positive intraoperative PLC had a significantly higher recurrent/metastatic rate (80\%) compared to those in the negative PLC group $(25 \%)(p<0.001$; Fig. 1$)$. The prognosis of patients with positive intraoperative PLC was worse than that of patients with negative PLC, with $60 \%$ and $12 \%$ of mortality rate respectively ( $p<$ 0.001; Fig.2). The median follow-up time was 53 months. (IQR: 33-66 months) We also separately analysed the recurrent/metastatic rate and survival based on the results of PLC. The patient with either PLC 1, 2 or 3 positive was significantly associated with the increasing recurrent rate and the decreasing survival rate, compared to those in the negative PLC group $(p<0.01)$. Patients with PLC 1 positive had the worst prognosis (Fig. 3$)$. The association between PLC status and patient baseline characteristics and clinicopathological characteristics were shown in Table 3. Positive intraoperative pleural lavage cytology was significantly associated with visceral pleural invasion $(p=0.018)$, lymphatic invasion $(p=0.043)$, and pathological stage $(p=0.003)$.

In our sub-group analysis, we analysed the association between intraoperative pleural lavage cytologic results and clinical outcomes for each pathological stage. We found that in pathological stage $1 \mathrm{~A}$ and 1B, patients with positive PLC had significantly higher recurrent/metastatic rate compared to those with negative $\mathrm{PLC}(p<0.05$; Table 4). In addition, patients in pathological stage 1B with positive PLC had both higher recurrent rate and worse survival rate compared to patients with negative PLC ( $p<0.05$; Table 5) However, the recurrent/metastatic rate and survival rate were not significantly different between two patient groups in other pathological stages.

\section{Discussion}

Back in 1958, the first report about cytological examination for cancer cells in pleural cavities from pleural lavage at the time of thoracotomy was published [14]. Later, Kondo et al [15] and Okumura et al [16] analysed the clinical significance of intraoperative pleural lavage cytology and reported its prognostic value. Pleural lavage cytology has been proved to be associated with the degree of pleural extension, microscopic pleural dissemination, pathologic stage, and the presence of lymphatic or vascular invasion in these studies. In 2014, Hokka et al [17] published a report showing a prognostic value of pleural lavage cytology in NSCLC patients with stage 1 disease. In our study, we compiled a five-year collection of data in Ramathibodi Hospital with the aim to provide the information about the clinical significance of intraoperative PLC status in NSCLC patients. The yield of positive intraoperative PLC was $11.2 \%$. This percentage is comparable with other previous studies $[16,18]$. For primary outcomes, we compared the recurrent/metastatic and survival rate between positive and negative PLC groups. The results suggested that a positive PLC status is significantly associated with poor clinical outcomes: the increasing recurrent rate and the decreasing survival rate. Apart fromthe recurrent/metastatic andsurvival rate, PLC status is significantly associated with visceral pleural invasion, vascular invasion and lymphatic invasion. Patients with positive PLC also seemed to have a higher pathological staging. Moreover, from our sub-group analysis, NSCLC patients with pathological stage 1A and 1B, which current recommendation suggests 
surveillance follow up without any adjuvant therapy [19], were found to have a statistically significant association between positive PLC status and their recurrent and survival rate. Since intraoperative pleural lavage is a noninvasive procedure and requires only simple equipment and a cytological examination, it can be easily added on to the usual operations. Besides, we recommend intraoperative pleural lavage cytologic status to be considered as one of the prognostic factors for further treatment planning and surveillance strategy, especially in NSCLC patients with pathological stage $1 \mathrm{~A}$ and $1 \mathrm{~B}$.

\section{Conclusion}

The PLC status was positive in $11.23 \%$ of NSCLC patients who underwent lungresection surgery. The positive finding significantly associated with higher rates of visceral pleural invasion, vascular invasion, lymphatic invasion, and a higher pathological staging. More importantly, a positive PLC status was significantly associated with higher recurrent/metastatic rate and worse survival rate, particularly for patients with early-stage diseases.

\section{Abbreviations}

IPL: Intraoperative pleural lavage

IQR: Interquartile range

NSCLC: Non-small cell lung cancer

PLC: Pleural lavage cytology

SD: Standard deviation

\section{Declarations}

\section{Ethics approval and consent to participate}

This study was approved by the Institutional Review Board of Faculty of Medicine Ramathibodi Hospital, Mahidol University and compliance with Helsinki declaration. (Certificate no. MURA2017/695; ID 10-60-19). The need of informed consent was waived by ethical committee of Faculty of Medicine Ramathibodi Hospital, Mahidol University, Thailand. Study protocol was in compliance with Helsinki declaration.

\section{Consent for publication}

Not applicable

\section{Availability of data and materials}

The datasets used and/or analysed during the current study available from the corresponding author on reasonable request.

\section{Competing interests}

The authors declare that they have no competing interests. 
Funding

None.

Author contribution

All authors (SE, PP and MN) have read and approved the manuscript. SE and MN conceptualized the study. SE conducted all of the data collection. SE and PP analysed the data. SE and MN wrote the first draft. SE and PP conducted reviewing and editing until the final version. MN was the supervisor of this study.

\section{Acknowledgements}

Not applicable

\section{References}

1. Sriplung H, Wiangnon S, Sontipong S, Sumitsawan Y, Martin N: Cancer incidence trends in Thailand, 19892000. Asian Pacific Journal of Cancer Prevention 2006, 7(2):239.

2. Vatanasapt V, Martin N, Sriplung H, Chindavijak K, Sontipong S, Sriamporn H, Parkin D, Ferlay J: Cancer incidence in Thailand, 1988-1991. Cancer Epidemiology and Prevention Biomarkers 1995, 4(5):475-483.

3. Virani S, Bilheem S, Chansaard W, Chitapanarux I, Daoprasert K, Khuanchana S, Leklob A, Pongnikorn D, Rozek LS, Siriarechakul S et al: National and Subnational Population-Based Incidence of Cancer in Thailand: Assessing Cancers with the Highest Burdens. Cancers (Basel) 2017, 9(8).

4. Goldstraw P, Crowley J, Chansky K, Giroux DJ, Groome PA, Rami-Porta R, Postmus PE, Rusch V, Sobin L, Committee IAftSoLCIS: The IASLC Lung Cancer Staging Project: proposals for the revision of the TNM stage groupings in the forthcoming (seventh) edition of the TNM Classification of malignant tumours. Journal of thoracic oncology 2007, 2(8):706-714.

5. Froudarakis ME: Pleural effusion in lung cancer: more questions than answers. Respiration 2012, 83(5):367376.

6. Morgensztern D, Waqar S, Subramanian J, Trinkaus K, Govindan R: Prognostic impact of malignant pleural effusion at presentation in patients with metastatic non-small-cell lung cancer. Journal of Thoracic Oncology 2012, 7(10):1485-1489.

7. Okada M, Tsubota N, Yoshimura M, Miyamoto Y, Maniwa Y: Role of pleural lavage cytology before resection for primary lung carcinoma. Annals of surgery 1999, 229(4):579.

8. Okada M, Sakamoto T, Nishio W, Uchino K, Tsuboshima K, Tsubota N: Pleural lavage cytology in non-small cell lung cancer: lessons from 1000 consecutive resections. The Journal of thoracic and cardiovascular surgery 2003, 126(6):1911-1915.

9. Kjellberg SI, Dresler CM, Goldberg M: Pleural cytologies in lung cancer without pleural effusions. The Annals of thoracic surgery 1997, 64(4):941-944.

10. Riquet M, Badoual C, Barthes FLP, Lhote F-M, Souilamas R, Hubsch J-P, Danel C: Visceral pleura invasion and pleural lavage tumor cytology by lung cancer: a prospective appraisal. The Annals of thoracic surgery 2003, 75(2):353-355. 
11. Lim E, Ali A, Theodorou P, Nicholson AG, Ladas G, Goldstraw P: Intraoperative pleural lavage cytology is an independent prognostic indicator for staging non-small cell lung cancer. The Journal of thoracic and cardiovascular surgery 2004, 127(4):1113-1118.

12. Shintani Y, Ohta M, Iwasaki T, Ikeda N, Kanou T, Tomita E, Nakagawa K, Yasumitsu T, Ohno Y: Intraoperative pleural lavage cytology after lung resection as an independent prognostic factor for staging lung cancer. The Journal of thoracic and cardiovascular surgery 2009, 137(4):835-839.

13. Detterbeck FC, Boffa DJ, Kim AW, Tanoue LT: The eighth edition lung cancer stage classification. Chest 2017, 151(1):193-203.

14. Spjut HJ, Hendrix VJ, Ramirez GA, Roper CL: Carcinoma cells in pleural cavity washings. Cancer 1958, 11(6):1222-1225.

15. Kondo H, Asamura H, Suemasu K, Goya T, Tsuchiya R, Naruke T, Yamagishi K, Uei Y: Prognostic significance of pleural lavage cytology immediately after thoracotomy in patients with lung cancer. The Journal of thoracic and cardiovascular surgery 1993, 106(6):1092-1097.

16. Okumura M, Ohshima S, Kotake Y, Morino H, Kikui M, Yasumitsu T: Intraoperative pleural lavage cytolopgy in lung cancer patients. The Annals of thoracic surgery 1991, 51(4):599-604.

17. Hokka D, Uchino K, Tane K, Ogawa H, Tane S, Tanaka Y, Tauchi S, Nishio W, Yoshimura M, Maniwa Y: Pleural lavage cytology as an independent prognostic factor in non-small-cell lung cancer patients with stage I disease and adenocarcinoma. Molecular and clinical oncology 2015, 3(1):244-248.

18. Collaborators IPLC: Impact of positive pleural lavage cytology on survival in patients having lung resection for non-small-cell lung cancer: an international individual patient data meta-analysis. The Journal of thoracic and cardiovascular surgery 2010, 139(6):1441-1446.

19. Postmus P, Kerr K, Oudkerk M, Senan S, Waller D, Vansteenkiste J, Escriu C, Peters S: Early and locally advanced non-small-cell lung cancer (NSCLC): ESMO Clinical Practice Guidelines for diagnosis, treatment and follow-up. Annals of Oncology 2017, 28(suppl_4):iv1-iv21.

\section{Tables}

Table 1. Patient baseline characteristic compare between non-recurrent and recurrent group. 


\begin{tabular}{|c|c|c|c|c|c|c|}
\hline \multirow[t]{2}{*}{ Data } & \multirow{2}{*}{$\begin{array}{l}\text { Total } \\
(n=178)\end{array}$} & \multirow{2}{*}{$\begin{array}{l}\begin{array}{l}\text { Non- } \\
\text { recurrent }\end{array} \\
(n=122)\end{array}$} & \multirow{2}{*}{$\begin{array}{l}\text { Recurrent } \\
(n=56)\end{array}$} & \multirow{2}{*}{$\begin{array}{l}\text { p- } \\
\text { value }\end{array}$} & \multicolumn{2}{|l|}{ Univariate } \\
\hline & & & & & $\mathrm{HR}(95 \% \mathrm{Cl})$ & $\begin{array}{l}\mathrm{p}- \\
\text { value }\end{array}$ \\
\hline \multicolumn{7}{|l|}{ Gender } \\
\hline Male & $67(37.64)$ & $42(34.43)$ & $25(44.64)$ & 0.191 & 1 & \\
\hline female & $111(62.36)$ & $80(65.57)$ & $31(55.36)$ & & $\begin{array}{l}0.676(0.39- \\
1.14)\end{array}$ & 0.146 \\
\hline Age(year), median(IQR) & $65(59,72)$ & $65(61,72)$ & $\begin{array}{l}63(56.5 \\
72.5)\end{array}$ & 0.279 & $\begin{array}{l}0.990(0.96- \\
1.02)\end{array}$ & 0.458 \\
\hline $\mathrm{BMI}$, mean $\pm \mathrm{SD}$ & $24.13+3.81$ & $24.25+3.53$ & $23.87+4.39$ & 0.536 & $\begin{array}{l}0.981(0.91- \\
1.05)\end{array}$ & 0.618 \\
\hline $\mathrm{DM}, \mathrm{n}(\%)$ & $32(17.98)$ & $21(17.21)$ & 11(19.64) & 0.695 & $\begin{array}{l}1.126(0.58- \\
2.17)\end{array}$ & 0.724 \\
\hline$H T, n(\%)$ & $92(51.69)$ & $67(54.92)$ & $25(44.64)$ & 0.203 & $\begin{array}{l}0.701(0.41- \\
1.18)\end{array}$ & 0.187 \\
\hline DLP, n(\%) & $69(38.76)$ & 18(32.14) & $51(41.80)$ & 0.219 & $\begin{array}{l}0.673(0.38- \\
1.18)\end{array}$ & 0.168 \\
\hline Other, n(\%) & $68(38.20)$ & 48(39.34) & 20(35.71) & 0.643 & & \\
\hline History of lung disease, $n(\%)$ & $34(19.10)$ & 23(18.85) & 11(19.64) & 0.901 & $\begin{array}{l}1.060(0.54- \\
2.05)\end{array}$ & 0.862 \\
\hline Never smoke, $\mathrm{n}(\%)$ & 127(71.35) & $90(73.77)$ & $37(66.07)$ & 0.291 & $\begin{array}{l}0.722(0.41- \\
1.25)\end{array}$ & 0.25 \\
\hline Current smoking, n(\%) & $15(8.43)$ & $11(9.02)$ & $4(7.14)$ & 0.676 & $\begin{array}{l}0.801(0.28- \\
2.20)\end{array}$ & 0.671 \\
\hline \multicolumn{7}{|l|}{ Incision, $n(\%) n=174$} \\
\hline Thoracotomy & $100(57.47)$ & $60(50.42)$ & $40(72.73)$ & 0.006 & 1 & \\
\hline VATS & $74(42.53)$ & $59(49.58)$ & $15(27.27)$ & & $\begin{array}{l}0.467(0.25- \\
0.84)\end{array}$ & 0.012 \\
\hline LOH(day), median(IQR) & $6.50(5,8)$ & $6(5,8)$ & $7(6,9)$ & 0.173 & & \\
\hline \multicolumn{7}{|l|}{ Vascular invasion, $n(\%) n=177$} \\
\hline Negative & 138(77.97) & 103(85.12) & $35(62.50)$ & 0 & 1 & \\
\hline Positive & $39(22.03)$ & $18(14.88)$ & $21(37.50)$ & & $\begin{array}{l}3.013(1.74- \\
5.19)\end{array}$ & 0 \\
\hline \multicolumn{7}{|l|}{$\begin{array}{l}\text { Visceral pleural invasion, } n(\%) \\
n=177\end{array}$} \\
\hline Negative & 135(76.27) & 102(84.30) & $33(58.93)$ & 0 & 1 & \\
\hline Positive & $42(23.73)$ & 19(15.70) & $23(41.07)$ & & $\begin{array}{l}2.958(1.73- \\
5.05)\end{array}$ & 0 \\
\hline
\end{tabular}


Lymphatic invasion, $\mathrm{n}(\%)$

$n=176$

$\begin{array}{lllllll}\text { Negative } & 151(85.80) & 114(95.00) & 37(66.07) & 0 & 1 \\ \text { Positive } & 25(14.20) & 6(5.00) & 19(33.93) & & 5.446(3.10- & 0\end{array}$

Cytology-Specimen1, n(\%)

$\begin{array}{lllllll}\text { Negative } & 167(93.82) & 122(100) & 45(80.36) & 0 & 1 \\ \text { Positive } & 11(6.18) & 0 & 11(19.64) & & \begin{array}{l}7.556(3.85- \\ 14.82)\end{array} & 0\end{array}$

Cytology-Specimen2, n(\%)

$\begin{array}{lllllll}\text { Negative } & 165(92.70) & 119(97.54) & 46(82.14) & 0 & 1 \\ \text { Positive } & 13(7.30) & 3(2.46) & 10(17.86) & & \begin{array}{l}4.649(2.31- \\ 9.34)\end{array}\end{array}$

Cytology-Specimen3, n(\%)

$\begin{array}{lllllll}\text { Negative } & 167(93.82) & 119(97.54) & 48(85.71) & 0.005 & 1 & \\ \text { Positive } & 11(6.18) & 3(2.46) & 8(14.29) & & \begin{array}{l}3.811(1.78- \\ 8.14)\end{array} & 0.001\end{array}$

Cytology-Specimen all, n(\%)

$\begin{array}{llllllll} & \text { Negative } & 158(88.76) & 118(96.72) & 40(71.43) & 0 & 1 & \\ & \text { positive } & 20(11.24) & 4(3.28) & 16(28.57) & & 5.545(3.05- & 0 \\ & & & & & & 10.07) & \\ & & 2.6(1.8, & 2.3(1.7, & 3.5(2.5, & 0 & 1.243(1.09- & 0.001 \\ \begin{array}{l}\text { Tumor } \\ \text { size }(\mathrm{cm}),\end{array} & & 3.6) & 3.2) & 5.0) & & 1.41) & \end{array}$

Pathological Staging (post-op), $n(\%)$

\begin{tabular}{|c|c|c|c|c|c|c|c|}
\hline & $1 \mathrm{~A}$ & $91(51.70)$ & $79(65.29)$ & $12(21.82)$ & 0 & 1 & \\
\hline & $1 \mathrm{~B}$ & $42(23.86)$ & $28(23.14)$ & $14(25.45)$ & & $\begin{array}{l}2.731(1.26- \\
5.91)\end{array}$ & 0.011 \\
\hline & $2 \mathrm{~A}$ & 16(9.09) & $5(4.13)$ & $11(20.00)$ & & $\begin{array}{l}8.969(3.94- \\
20.40)\end{array}$ & 0 \\
\hline & $2 B$ & $12(6.82)$ & $4(3.31)$ & $8(14.55)$ & & $\begin{array}{l}7.280(2.95- \\
17.95)\end{array}$ & 0 \\
\hline & $3 A$ & $12(6.82)$ & $3(2.48)$ & $9(16.36)$ & & $\begin{array}{l}11.22(4.70- \\
26.77)\end{array}$ & 0 \\
\hline Death, n(\%) & & $32(17.98)$ & $3(2.46)$ & $29(51.79)$ & 0 & $\begin{array}{l}10.365(6.05- \\
17.75)\end{array}$ & 0 \\
\hline
\end{tabular}

Table 2. Patient baseline characteristic compare between non-death and death group. 


\begin{tabular}{|c|c|c|c|c|c|c|}
\hline \multirow[t]{2}{*}{ Data } & \multirow{2}{*}{$\begin{array}{l}\text { Total } \\
(n=178)\end{array}$} & \multirow{2}{*}{$\begin{array}{l}\text { Non-death } \\
(n=152)\end{array}$} & \multirow{2}{*}{$\begin{array}{l}\text { Death } \\
(n=32)\end{array}$} & \multirow{2}{*}{$\begin{array}{l}\text { p- } \\
\text { value }\end{array}$} & \multicolumn{2}{|l|}{ Univariate } \\
\hline & & & & & $\mathrm{HR}(95 \% \mathrm{Cl})$ & $\begin{array}{l}\mathrm{p}- \\
\text { value }\end{array}$ \\
\hline \multicolumn{7}{|l|}{ Gender } \\
\hline Male & $67(37.64)$ & $51(34.93)$ & $16(50.00)$ & 0.111 & 1 & \\
\hline female & $111(62.36)$ & $95(65.07)$ & $16(50.00)$ & & $\begin{array}{l}0.565(0.28- \\
1.13)\end{array}$ & 0.107 \\
\hline Age(year), median(IQR) & $65(59,72)$ & $65(60,72)$ & $\begin{array}{l}\text { 64.5(54, } \\
73.5)\end{array}$ & 0.551 & $\begin{array}{l}0.991(0.95- \\
1.02)\end{array}$ & 0.622 \\
\hline $\mathrm{BMI}$, mean \pm SD & $24.13+3.81$ & $24.09+3.56$ & $24.32+4.86$ & 0.755 & $\begin{array}{l}1.011(0.91- \\
1.12)\end{array}$ & 0.823 \\
\hline $\mathrm{DM}, \mathrm{n}(\%)$ & $32(17.98)$ & $28(19.18)$ & $4(12.50)$ & 0.373 & $\begin{array}{l}0.698(0.24- \\
1.99)\end{array}$ & 0.502 \\
\hline $\mathrm{HT}, \mathrm{n}(\%)$ & $92(51.69)$ & $79(54.11)$ & $13(40.63)$ & 0.167 & $\begin{array}{l}0.601(0.29- \\
1.21)\end{array}$ & 0.158 \\
\hline DLP, n(\%) & $69(38.76)$ & $59(40.41)$ & $10(31.25)$ & 0.335 & $\begin{array}{l}0.656(0.31- \\
1.38)\end{array}$ & 0.27 \\
\hline Other, $\mathrm{n}(\%)$ & $68(38.20)$ & $52(35.62)$ & $16(50.00)$ & 0.129 & & \\
\hline History of lung disease, $n(\%)$ & $34(19.10)$ & $30(20.55)$ & $4(12.50)$ & 0.294 & $\begin{array}{l}0.674(0.23- \\
1.92)\end{array}$ & 0.462 \\
\hline Never smoke, n(\%) & 127(71.35) & 106(72.60) & $21(65.63)$ & 0.429 & $\begin{array}{l}0.716(0.34- \\
1.48)\end{array}$ & 0.371 \\
\hline Current smoking, $n(\%)$ & $15(8.43)$ & 12(8.33) & $3(9.68)$ & 0.732 & $\begin{array}{l}1.145(0.34- \\
3.77)\end{array}$ & 0.823 \\
\hline \multicolumn{7}{|l|}{ Incision, $n(\%) n=174$} \\
\hline Thoracotomy & $100(57.47)$ & $79(54.86)$ & $21(70.00)$ & 0.127 & 1 & \\
\hline VATS & $74(42.53)$ & $65(45.14)$ & $9(30.00)$ & & $\begin{array}{l}0.682(0.31- \\
1.49)\end{array}$ & 0.339 \\
\hline LOH(day), median(IQR) & $6.50(5,8)$ & $6(5,8)$ & $7.5(6,9)$ & 0.046 & & \\
\hline \multicolumn{7}{|l|}{ Vascular invasion, $n(\%) n=177$} \\
\hline Negative & 138(77.97) & $120(82.76)$ & $18(56.25)$ & 0.001 & 1 & \\
\hline positive & $39(22.03)$ & $25(17.24)$ & $14(43.75)$ & & $\begin{array}{l}4.187(2.06- \\
8.48)\end{array}$ & 0 \\
\hline \multicolumn{7}{|l|}{$\begin{array}{l}\text { Visceral pleural invasion, } n(\%) \\
n=177\end{array}$} \\
\hline Negative & $135(76.27)$ & 118(81.38) & $17(53.13)$ & 0.001 & 1 & \\
\hline positive & $42(23.73)$ & $27(18.62)$ & $15(46.88)$ & & $\begin{array}{l}4.009(1.99- \\
8.07)\end{array}$ & 0 \\
\hline
\end{tabular}


$n=176$

\begin{tabular}{lllllll} 
Negative & $151(85.80)$ & $134(93.06)$ & $17(53.13)$ & 0 & 1 \\
\hline positive & $25(14.20)$ & $10(6.94)$ & $15(46.88)$ & & $\begin{array}{l}8.572(4.25- \\
17.27)\end{array}$ & 0
\end{tabular}

Cytology-Specimen 1, n(\%)

$\begin{array}{lllllll}\text { Negative } & 167(93.82) & 143(97.95) & 24(75.00) & 0 & 1 \\ \text { positive } & 11(6.18) & 3(2.05) & 8(25.00) & & \begin{array}{l}6.897(3.08- \\ 15.43)\end{array}\end{array}$

Cytology-Specimen 2, n(\%)

$\begin{array}{lllllll}\text { Negative } & 165(92.70) & 141(96.58) & 24(75.00) & 0 & 1 \\ \text { Positive } & 13(7.30) & 5(3.42) & 8(25.00) & & 12.29(5.05- & 0\end{array}$

Cytology-Specimen 3, n(\%)

$\begin{array}{lllll}\text { Negative } & 167(93.82) & 141(96.58) & 26(81.25) & 0.005 \\ \text { Positive } & 11(6.18) & 5(3.42) & 6(18.75) & \end{array}$

9.219(3.51- 0 24.21)

Cytology-Specimen all, n(\%)

\begin{tabular}{|c|c|c|c|c|c|c|}
\hline Negative & 158(88.76) & $138(94.52)$ & $20(62.50)$ & 0 & 1 & \\
\hline \multirow[t]{2}{*}{ positive } & $20(11.24)$ & $8(5.48)$ & $12(37.50)$ & & $\begin{array}{l}8.903(4.25- \\
18.64)\end{array}$ & 0 \\
\hline & $\begin{array}{l}2.6(1.8 \\
3.6)\end{array}$ & $\begin{array}{l}2.5(1.8 \\
3.5)\end{array}$ & $\begin{array}{l}3.0(2.5 \\
5.0)\end{array}$ & 0.01 & $\begin{array}{l}1.153(0.94- \\
1.40)\end{array}$ & 0.152 \\
\hline
\end{tabular}

Tumor

size $(\mathrm{cm})$,

median(IQR)

Pathological Staging(post-op),

$\mathrm{n}(\%)$

\begin{tabular}{|c|c|c|c|c|c|c|}
\hline $1 \mathrm{~A}$ & $91(51.70)$ & $87(60.42)$ & $4(12.50)$ & 0 & 1 & \\
\hline $1 \mathrm{~B}$ & $42(23.86)$ & $36(25.00)$ & $6(18.75)$ & & $\begin{array}{l}3.415(0.96- \\
12.11)\end{array}$ & 0.057 \\
\hline $2 \mathrm{~A}$ & 16(9.09) & $7(4.86)$ & $9(28.13)$ & & $\begin{array}{l}15.576(4.79- \\
50.61)\end{array}$ & 0 \\
\hline $2 B$ & $12(6.82)$ & $7(4.86)$ & $5(15.63)$ & & $\begin{array}{l}15.67(4.11- \\
59.79)\end{array}$ & 0 \\
\hline $3 A$ & $12(6.82)$ & $5(3.47)$ & $7(21.88)$ & & $\begin{array}{l}28.14(8.10- \\
97.81)\end{array}$ & 0 \\
\hline
\end{tabular}

Complication, $\mathrm{n}(\%)$

AF, hepatitis, $\quad 1$

bronchopleural

fistula

PE

1

0

3(2.05) 3(9.38)

0.038

$0 \quad 1$

1

Page $11 / 17$ 


\begin{tabular}{|c|c|c|c|c|}
\hline air-leak & 3 & 2 & 1 & \\
\hline stroke & 1 & 1 & 0 & \\
\hline Recurence, n(\%) & $56(31.46)$ & $27(18.49)$ & $29(90.63)$ & 0 \\
\hline
\end{tabular}

Table 3. Associations between pleural lavage cytology status and patient's clinicopathological characteristics. 


\begin{tabular}{|c|c|c|c|c|}
\hline Data & total & Cyto-Neg & Cyto-Pos & p-value \\
\hline & $(n=178)$ & $(n=122)$ & $(n=56)$ & \\
\hline \multicolumn{5}{|l|}{ Gender } \\
\hline Male & $67(37.64)$ & $59(37.34)$ & $8(40.00)$ & 0.817 \\
\hline female & $111(62.36)$ & $99(62.66)$ & $12(60.00)$ & \\
\hline Age(year), median(IQR) & $65(59,72)$ & $65(59,72)$ & $66(57,74.5)$ & 0.646 \\
\hline BMI, mean $\pm S D$ & $24.13 \pm 3.81$ & $23.97 \pm 3.56$ & $25.39 \pm 5.36$ & 0.26 \\
\hline $\mathrm{DM}, \mathrm{n}(\%)$ & $32(17.98)$ & $27(17.09)$ & $5(25.00)$ & 0.385 \\
\hline$H T, n(\%)$ & $92(51.69)$ & $80(50.63)$ & $12(60.00)$ & 0.43 \\
\hline DLP, n(\%) & $69(38.76)$ & $61(38.61)$ & $8(40.00)$ & 0.904 \\
\hline Other, n(\%) & $68(38.20)$ & $59(37.34)$ & $9(45.00)$ & 0.507 \\
\hline History of lung disease, $n(\%)$ & $34(19.10)$ & $31(19.62)$ & $3(15.00)$ & 0.769 \\
\hline Never smoke, $\mathrm{n}(\%)$ & $127(71.35)$ & $109(68.99)$ & 18(90.00) & 0.065 \\
\hline Current smoking, n(\%) & $15(8.43)$ & 15(9.62) & 0 & 0.377 \\
\hline \multicolumn{5}{|l|}{ Incision, $n(\%) n=174$} \\
\hline Thoracotomy & $100(57.47)$ & $88(57.14)$ & $12(60.00)$ & 0.808 \\
\hline VATS & $74(42.53)$ & $66(42.86)$ & $8(40.00)$ & \\
\hline LOH(day), median(IQR) & $6.50(5,8)$ & $7(5,8)$ & $6(5,9)$ & 0.899 \\
\hline \multicolumn{5}{|l|}{ Vascular invasion, $n(\%) n=177$} \\
\hline Negative & 138(77.97) & $125(79.62)$ & $13(65.00)$ & 0.137 \\
\hline Positive & $39(22.03)$ & $32(20.38)$ & $7(35.00)$ & \\
\hline \multicolumn{5}{|c|}{ Visceral pleura invasion, $n(\%) n=177$} \\
\hline Negative & 135(76.27) & 124(78.98) & $11(55.00)$ & 0.018 \\
\hline Positive & $42(23.73)$ & $33(21.02)$ & $9(45.00)$ & \\
\hline \multicolumn{5}{|l|}{ Lymphatic invasion, $n(\%) n=176$} \\
\hline Negative & $151(85.80)$ & $137(87.82)$ & $14(70.00)$ & 0.043 \\
\hline Positive & $25(14.20)$ & 19(12.18) & $6(30.00)$ & \\
\hline \multicolumn{5}{|c|}{ Pathological Staging(post-op), n(\%) } \\
\hline $1 \mathrm{~A}$ & $91(51.70)$ & $85(54.49)$ & $6(30.00)$ & 0.003 \\
\hline 1B & $42(23.86)$ & $37(23.72)$ & $5(25.00)$ & \\
\hline $2 \mathrm{~A}$ & 16(9.09) & $16(10.26)$ & 0 & \\
\hline
\end{tabular}




\begin{tabular}{|cllll|}
\hline $2 B$ & $12(6.82)$ & $7(4.49)$ & $5(25.00)$ & \\
\hline $3 A$ & $12(6.82)$ & $8(5.13)$ & $4(20.00)$ & \\
\hline Death, $\mathrm{n}(\%)$ & $32(17.98)$ & $20(12.66)$ & $12(60.00)$ & 0 \\
\hline Recurrence, $\mathrm{n}(\%)$ & $56(31.46)$ & $40(25.32)$ & $16(80.00)$ & 0 \\
\hline
\end{tabular}

Table 4 Recurrent/metastatic in patients with pathological stage $1 \mathrm{~A}$ and $1 \mathrm{~B}$ comparing between positive and negative PLC result. The $\mathrm{P}$ value was determined using Fisher' exact test

\begin{tabular}{|ccclll|}
\hline Pathological stage & Total & Non-recurrent & Recurrent/metastasis & p-value \\
\hline 1A & Cytology positive & 6 & 3 & 3 & 0.28 \\
\cline { 2 - 7 } & Cytology negative & 85 & 76 & 9 & \\
\hline 1B & Cytology positive & 5 & 0 & 5 & 0.002 \\
\cline { 2 - 7 } & Cytology negative & 37 & 28 & 9 & \\
\hline
\end{tabular}

Table 5 Overall Survival in patients with pathological stage $1 \mathrm{~A}$ and $1 \mathrm{~B}$ comparing between positive and negative PLC result. The $\mathrm{P}$ value was determined using Fisher' exact test

\begin{tabular}{|cccccc|}
\hline \multicolumn{2}{|l}{ Pathological stage } & Total & Alive & Death & p-value \\
1A & Cytology positive & 6 & 5 & 1 & 0.242 \\
\cline { 2 - 7 } & Cytology negative & 85 & 82 & 3 & \\
1B & Cytology positive & 5 & 2 & 3 & 0.015 \\
\cline { 2 - 7 } & Cytology negative & 37 & 34 & 3 & \\
\hline
\end{tabular}

\section{Figures}




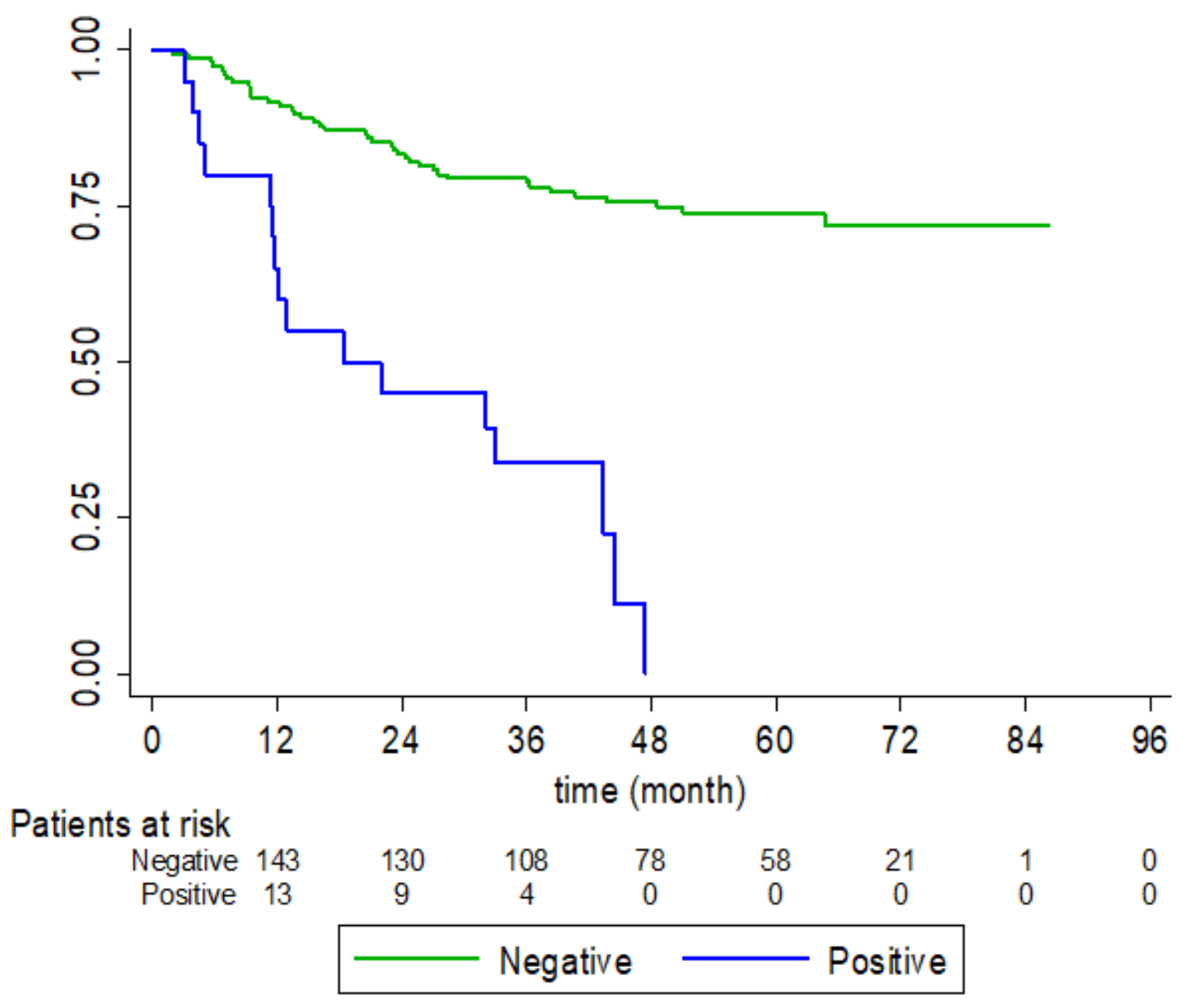

\section{Figure 1}

Kaplan-Meier plot of recurrent/metastatic rate in 178 patients with NSCLC and its association with the PLC result. The $P$ value was determined using the log-rank test. 


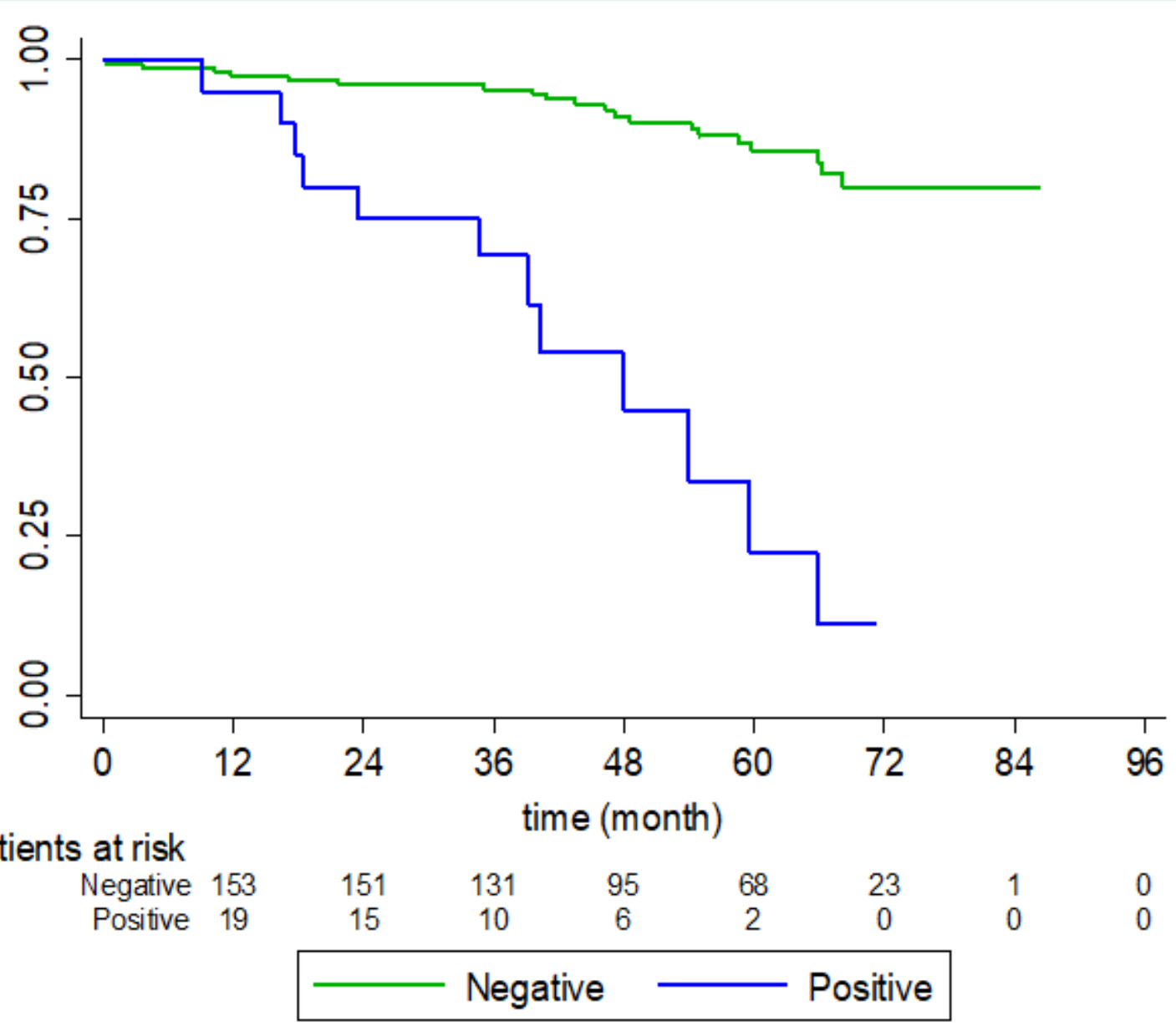

Figure 2

Kaplan-Meier plot of overall survival in 178 patients with NSCLC and its association with the PLC result. The P value was determined using the log-rank test. 


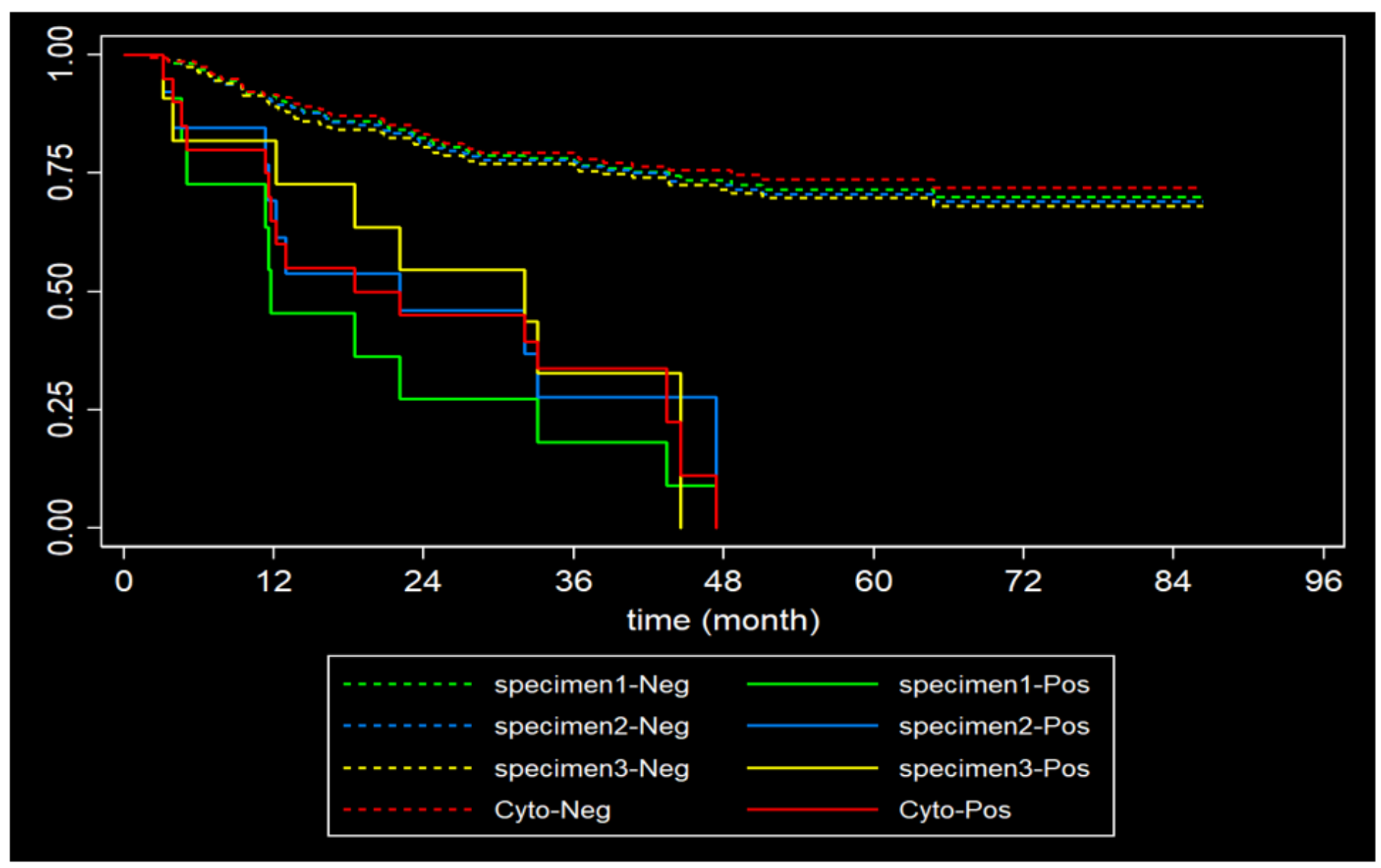

Figure 3

Kaplan-Meier plot of recurrent/metastatic rate in 178 patients with NSCLC and its association with the PLC result divided by type of specimen. The $P$ value was determined using the log-rank test. 